\title{
Outcomes and Predictors of 30-Day Readmissions for Hyperthyroidism: A Nationwide Study
}

\author{
Michael Salim ${ }^{1}$, Zain El-amir², Asim Kichloo ${ }^{2,3}$, Farah Wani ${ }^{3}$, Ehizogie Edigin ${ }^{4}$, Hafeez Shaka ${ }^{4}$ \\ ${ }^{1}$ Department of Internal Medicine, Mount Sinai Hospital, Chicago, IL; ${ }^{2}$ Department of Internal Medicine, Central Michigan \\ University, Saginaw, MI; ${ }^{3}$ Department of Medicine, Samaritan Medical Center, Watertown, NY; ${ }^{4}$ Department of Internal \\ Medicine, John H. Stroger, Jr. Hospital of Cook County, Chicago, IL, USA
}

Hyperthyroidism is associated with an elevated risk of cardiovascular events and worse hospital outcomes. The Nationwide Readmissions Database (NRD) 2018 was used to determine the characteristics of 30-day readmission in patients with hyperthyroidism. The 30-day all-cause readmission rate for hyperthyroidism was $10.3 \%$. About $21.7 \%$ had hyperthyroidism as the principal diagnosis on readmission. Readmissions were associated with an increased odds of inpatient mortality (odds ratio, 7.04; $95 \%$ confidence interval [CI], 3.97 to 12.49), length of stay (5.2 days vs. 4.0 days; $95 \%$ CI, 0.7 to 1.8), total hospital charges, and cost of hospitalizations. Independent predictors of 30-day all-cause readmissions included Charlson Comorbidity Index $\geq 3$ (adjusted hazard ratio [aHR], $1.76 ; 95 \% \mathrm{CI}, 1.15$ to 2.71 ), discharge against medical advice (aHR, 2.30; $95 \% \mathrm{CI}, 1.50$ to 3.53), protein-energy malnutrition (aHR, $1.54 ; 95 \% \mathrm{CI}, 1.15$ to 2.07 ), and atrial fibrillation (aHR, 1.41; $95 \% \mathrm{CI}, 1.11$ to 1.79 ). Aggressive but appropriate monitoring is warranted in patients with hyperthyroidism to prevent readmissions.

Keywords: Hyperthyroidism; Patient readmission; Hospital mortality

\section{INTRODUCTION}

Several studies have indicated a substantial increase in hospital cost, length of stay, and mortality in patients readmitted under 30 days for a variety of clinical conditions. Some of the risk factors for readmissions are, in fact, preventable and could improve hospital outcomes [1,2]. Hyperthyroidism is associated with an elevated risk of cardiovascular events and worse hospital outcomes $[3,4]$. Consequently, it is essential to understand more about the impacts of hyperthyroidism and other related factors on readmissions in order to prevent it. This study aims to analyze the impacts of hyperthyroidism on the readmission rate, the clinical outcomes, and predictors for readmissions.

Received: 15 July 2021, Revised: 30 September 2021,

Accepted: 21 October 2021

Corresponding author: Michael Salim

Department of Internal Medicine, Mount Sinai Hospital, 1500 S. Fairfield, Chicago, IL 60608, USA

Tel: +1-773-257-5077, Fax: +1-773-257-6027, E-mail: michael.salim@sinai.org

\section{METHODS}

\section{Ethical considerations}

The Nationwide Readmissions Database (NRD) database lacks patient identifiers. The Cook County Health Institutional Review Board deemed the research project exempt from approval since it is a retrospective review of already collected, de-identified data sets.

\section{Design and data source}

This was a retrospective cohort study involving adult hospitalizations for hyperthyroidism in the United States in 2018. The NRD for 2018 was the data source. The NRD is the largest pub-

Copyright $(2021$ Korean Endocrine Society

This is an Open Access article distributed under the terms of the Creative Commons Attribution Non-Commercial License (https://creativecommons.org/ licenses/by-nc/4.0/) which permits unrestricted non-commercial use, distribution, and reproduction in any medium, provided the original work is properly cited. 
licly available all-payer inpatient health care readmission database drawn from the Agency for Healthcare Research and Quality (AHRQ) Healthcare Cost and Utilization Project (HCUP) State Inpatient Databases (SID). The NRD 2018 contains discharge data for $58.7 \%$ of all United States hospitalizations. It includes both patient and hospital-level information. Up to 40 discharge diagnoses and 25 procedures are collected for each patient using the International Classification of Diseases, 10th Revision, Clinical Modification (ICD-10-CM/PCS). Hospitals are stratified according to ownership control, the number of beds, teaching status, urban/rural location, and geographic region. The NRD allows a weighted analysis to obtain 100\% of the United States admissions within a given year.

\section{Study population and definition}

The study involved hospitalizations from the NRD with hyperthyroidism as the principal diagnosis for index admission using ICD-10 codes (E05.X). Principal diagnoses and comorbidities were identified using the appropriate ICD-10 codes. NRD variables were used to identify each patient's age, sex, insurance type, median household income, hospital bed size, teaching status, length of hospital stay (LOS), and total hospital charges (THC). The cost of hospitalization $(\mathrm{COH})$ was obtained from the cost to charge ratio data provided by HCUP.

Hospitalizations with age less than 18 and elective hospitalizations were excluded. December hospitalizations were also excluded as they did not contain complete data to assess 30-day readmissions. Using unique hospitalization identifiers, index hospitalizations were identified, and one subsequent hospitalization within 30 days was tagged as readmission. Elective and traumatic hospitalizations were excluded from readmissions. Comorbidity burden was assessed using Sundararajan's adaptation of the modified Deyo's Charlson Comorbidity Index (CCI) [5].

\section{Outcome measures}

This study compared the groups of patients with index admission and patients with readmission. The primary outcome was the rate and reasons for 30-day readmission in patients with hyperthyroidism. Secondary outcomes included the mortality rate, LOS, THC, COH, and predictors of 30-day all-cause readmissions in patients with hyperthyroidism [6].

\section{Statistical analysis}

Stata version 16 software (StataCorp., College Station, TX, USA) was used for data analysis. All analyses were conducted using the weighted samples for national estimates in adjunct with HCUP regulations for using the NRD database. Comorbidities were calculated as proportions of the cohort, and the chisquare test was used to compare these characteristics between the index and readmissions. Univariable regression was used to compare readmission mortality, LOS, THC, and $\mathrm{COH}$. A univariable Cox regression analysis was performed to identify variables with hazard ratios for 30 -day readmission $<0.20$ to obtain confounders for readmission. Subsequently, a multivariable Cox regression analysis was done to identify independent predictors for readmissions with a $P<0.05$ set as the threshold for statistical significance. Confounder variables included in the final model were grouped age, insurance, and CCI, disposition, dyslipidemia, chronic obstructive pulmonary disease, diabetes mellitus, chronic kidney disease, obesity, protein-energy malnutrition, atrial fibrillation, and history of neoplasm.

\section{RESULTS}

\section{Baseline characteristics of index admissions and readmissions}

The patients in the readmission group were older than those in the index admission group (48.3 years vs. 53.6 years, $P<0.001$ ). There was no significant difference in sex distribution between both groups. Readmissions were more likely to have a CCI $\geq 3$ (29.0\% vs. $12.9 \%$ ) and Medicaid as the main insurer (27.3\% vs. $40.6 \%$ ). The readmission group also had higher proportion of patients with comorbid diabetes mellitus $(27.2 \%$ vs. $17.6 \%$, $P<0.001)$, congestive heart failure (23.0\% vs. $18.5 \%, P=0.025)$, chronic kidney disease $(10.6 \%$ vs. $6.6 \%, P=0.003)$, and atrial fibrillation $(27.0 \%$ vs. $32.0 \%, P=0.032)$ (Supplemental Table S1).

\section{Rate and etiologies for 30-day readmissions in hospitalizations with hyperthyroidism}

There were 6,979 index hospitalizations identified in the current study that met the inclusion criteria. Of these hospitalizations, there were 6,922 (99.2\%) patients discharged alive. The 30-day all-cause readmission rate for hyperthyroidism was $10.3 \%$. The highest proportion of readmissions was for hyperthyroidism $(21.7 \%)$, followed by paroxysmal atrial fibrillation $(6.6 \%)$, sepsis from unspecified organisms $(6.2 \%)$, hypertensive heart disease with heart failure (3.7\%), and acute renal failure unspecified $(2.4 \%)$ (Supplemental Table S2).

\section{Outcomes in the index admissions and readmissions}

Readmission following hyperthyroidism was associated with a significantly increased odds of inpatient mortality (5.5\% vs. 
$0.8 \%$; odds ratio [OR], 7.04; $95 \%$ confidence interval [CI], 3.97 to $12.49 ; P<0.001$ ), increased mean $\operatorname{LOS}(5.2$ days vs. 4.0 days; coefficient, $1.3 ; 95 \% \mathrm{CI}, 0.7$ to $1.8 ; P<0.001)$, and higher THC and $\mathrm{COH}$ (Table 1) when compared to the index admission.

\section{Predictors of 30-day all-cause readmissions of hyperthyroidism hospitalizations}

After adjusting for potential confounders, independent predictors of 30-day all-cause readmissions of hyperthyroidism included index hospitalizations with $\mathrm{CCI} \geq 3$ (adjusted hazard ratio [aHR], 1.76; $95 \% \mathrm{CI}, 1.15$ to $2.71 ; P=0.009$ ), discharge against medical advice (aHR, 2.30; 95\% CI, 1.50 to $3.53 ; P<0.001$ ), protein-energy malnutrition (aHR, $1.54 ; 95 \% \mathrm{CI}, 1.15$ to 2.07 ; $P=0.004$ ), and atrial fibrillation (aHR, $1.41 ; 95 \% \mathrm{CI}, 1.11$ to

\section{$1.79 ; P=0.004)($ Table 2).}

\section{DISCUSSION}

By analyzing the largest readmission database in the United States, we found that the 30-day all-cause readmission after hospitalization due to hyperthyroidism was $10.3 \%$. Several studies have attempted to identify approaches to decrease these readmissions, especially in patients undergoing thyroidectomy $[7,8]$.

The highest proportion of readmission diagnoses for patients with hyperthyroidism was hyperthyroidism, followed by paroxysmal atrial fibrillation, sepsis from unspecified organisms, and hypertensive heart disease with heart failure. Brito et al. [9] re-

Table 1. Clinical Outcomes in Hospitalizations with Hyperthyroidism

\begin{tabular}{|c|c|c|c|c|}
\hline Outcome & Index admission, $\%$ & 30-Day readmission, $\%$ & OR $(95 \% \mathrm{CI})$ & $P$ value $^{\mathrm{a}}$ \\
\hline In hospital mortality & 0.82 & 5.49 & $7.04(3.97-12.49)$ & $<0.001^{\mathrm{a}}$ \\
\hline Length of stay, mean & 4.0 & 5.2 & $1.3^{\mathrm{b}}(0.7-1.8)$ & $<0.001^{\mathrm{a}}$ \\
\hline Total hospital charges, mean US\$ & 39,565 & 56,154 & $16,590^{\mathrm{b}}(6,872-26,307)$ & $0.001^{\mathrm{a}}$ \\
\hline Total hospital cost, mean US\$ & 9,242 & 12,351 & $3,108^{b}(1,448-4,768)$ & $<0.001^{\mathrm{a}}$ \\
\hline
\end{tabular}

OR, odds ratio; CI, confidence interval.

${ }^{a}$ Statistically significant; ${ }^{b}$ Mean difference.

Table 2. Independent Predictors of 30-Day All-Cause Readmissions of Hyperthyroidism Hospitalizations

\begin{tabular}{|c|c|c|c|}
\hline Variable & aHR & $95 \% \mathrm{CI}$ & $P$ value $^{\mathrm{a}}$ \\
\hline Discharge against medical advice & 2.3 & $1.50-3.53$ & $<0.001^{\mathrm{a}}$ \\
\hline Charlson index of $\geq 3$ & 1.76 & $1.15-2.71$ & $0.009^{\mathrm{a}}$ \\
\hline Protein energy malnutrition & 1.54 & $1.15-2.07$ & $0.004^{\mathrm{a}}$ \\
\hline Atrial fibrillation & 1.41 & $1.11-1.79$ & $0.004^{\mathrm{a}}$ \\
\hline Charlson index of 2 & 1.4 & $0.92-2.13$ & 0.121 \\
\hline History of neoplasm & 1.34 & $0.87-2.07$ & 0.189 \\
\hline Chronic kidney disease & 1.32 & $0.90-1.94$ & 0.158 \\
\hline Obesity & 1.18 & $0.88-1.57$ & 0.272 \\
\hline Chronic obstructive pulmonary disease & 1.16 & $0.83-1.63$ & 0.383 \\
\hline Diabetes & 1.08 & $0.80-1.45$ & 0.625 \\
\hline Dyslipidemia & 1.02 & $0.77-1.36$ & 0.867 \\
\hline \multicolumn{4}{|l|}{ Age, $\mathrm{yr}^{\mathrm{b}}$} \\
\hline $40-64$ & 0.92 & $0.67-1.26$ & 0.601 \\
\hline$\geq 65$ & 0.98 & $0.64-1.50$ & 0.913 \\
\hline Private insurance $\mathrm{c}^{\mathrm{c}}$ & 0.56 & $0.38-0.82$ & $0.003^{\mathrm{a}}$ \\
\hline
\end{tabular}

aHR, adjusted hazard ratio; CI, confidence interval.

a Statistically significant; ${ }^{b}$ Relative to patients aged $18-39$ years; ' $R$ Relative to Medicaid. 
ported that in the United States, hyperthyroidism was mainly treated with antithyroid drug therapy $(60 \%)$, radioactive iodine $(33 \%)$, and thyroidectomy $(6 \%)$. However, the success rates of antithyroid drug therapy were only $50 \%$ for antithyroid drug therapy [9]. This might explain the high readmission rates of hyperthyroidism. Additionally, patients with hyperthyroidism treated with radioactive iodine had a higher risk of atrial fibrillation, cardiovascular disease-related hospitalization, and mortality $[10,11]$. Furthermore, hypothyroidism is a complication from radioactive iodine and thyroidectomy that could increase the risk of sepsis [12].

Readmission following hyperthyroidism was associated with significantly increased odds of inpatient mortality, mean LOS, THC, and $\mathrm{COH}$ compared to the index admissions. Hyperthyroidism has been shown to result in increased mortality, with one meta-analysis finding an increase in mortality of about $20 \%$ [13]. Being readmitted with hyperthyroidism, as was found to be the case in numerous patients in the readmission cohort, may reflect inadequate control of the condition and thus potentially increased mortality and morbidity [13].

In this study, independent predictors of 30-day all-cause readmissions of hyperthyroidism included index hospitalizations with higher CCI, discharge against medical advice, protein-energy malnutrition, and atrial fibrillation. Patients with these conditions would warrant closer follow-up to avoid readmissions $[14,15]$.

This study has limitations that should be noted. Data from the NRD are subject to biases that are associated with retrospective studies. The database reports information on hospitalizations rather than individual patients; therefore, patients admitted with numerous readmissions are included multiple times in the data set. The database also does not include information about the prescription medications, severity or control of hyperthyroidism, and intensive care unit stay at the time of admission or readmission. Finally, the NRD uses ICD-10 codes to report hospitalizations, making it subject to potential coding errors. Despite the limitations mentioned above, the large sample size, scientific inquiries, long study period, and analysis techniques enabled this study to shed light on hyperthyroidism readmissions while encouraging future large multicenter controlled prospective studies and further discourse on the topic.

In conclusion, patients with hyperthyroidism comorbidities (high CCI, malnutrition, and atrial fibrillation) have increased readmission rates and worsened clinical outcomes of readmissions. Thus, aggressive but appropriate monitoring may be warranted in patients with these conditions and hyperthyroidism.

\section{CONFLICTS OF INTEREST}

No potential conflict of interest relevant to this article was reported.

\section{AUTHOR CONTRIBUTIONS}

Conception or design: M.S., H.S. Acquisition, analysis, or interpretation of data: M.S., H.S. Drafting the work or revising: M.S., Z.E., A.K., F.W., E.E., H.S. Final approval of the manuscript: M.S., Z.E., A.K., F.W., E.E., H.S.

\section{ORCID}

Michael Salim https://orcid.org/0000-0002-1148-8940

\section{REFERENCES}

1. Bilal M, Abougergi MS, Tayyem O, Parupudi S, Rockey DC. Thirty-day readmission after esophageal variceal hemorrhage and its impact on outcomes in the United States. J Clin Gastroenterol 2020;54:477-83.

2. Boehme AK, Kulick ER, Canning M, Alvord T, Khaksari B, Omran S, et al. Infections increase the risk of 30-day readmissions among stroke survivors. Stroke 2018;49:29993005 .

3. Galindo RJ, Hurtado CR, Pasquel FJ, Garcia Tome R, Peng L, Umpierrez GE. National trends in incidence, mortality, and clinical outcomes of patients hospitalized for thyrotoxicosis with and without thyroid storm in the United States, 2004-2013. Thyroid 2019;29:36-43.

4. Luo B, Yu Z, Li Y. Thyroid hormone disorders and sepsis. Biomed Mater Eng 2017;28(s1):S237-41.

5. Sundararajan V, Quan H, Halfon P, Fushimi K, Luthi JC, Burnand $\mathrm{B}$, et al. Cross-national comparative performance of three versions of the ICD-10 Charlson index. Med Care 2007;45:1210-5.

6. Agency for Healthcare Research and Quality. HCUP user support (HCUP-US) [Internet]. Rockville: AHRQ; 2021 [cited 2021 Nov 16]. Available from: https://www.hcup-us.ahrq. gov.

7. Hsieh LB, Yen TW, Dream S, Patel D, Evans DB, Wang TS. Perioperative management and outcomes of hyperthyroid patients unable to tolerate antithyroid drugs. World J Surg 2020;44:3770-7.

8. Pesce CE, Shiue Z, Tsai HL, Umbricht CB, Tufano RP, 
Dackiw AP, et al. Postoperative hypocalcemia after thyroidectomy for Graves' disease. Thyroid 2010;20:1279-83.

9. Brito JP, Payne S, Singh Ospina N, Rodriguez-Gutierrez R, Maraka S, Sangaralingham LR, et al. Patterns of use, efficacy, and safety of treatment options for patients with Graves' disease: a nationwide population-based study. Thyroid 2020; 30:357-64.

10. Ryodi E, Metso S, Huhtala H, Valimaki M, Auvinen A, Jaatinen P. Cardiovascular morbidity and mortality after treatment of hyperthyroidism with either radioactive iodine or thyroidectomy. Thyroid 2018;28:1111-20.

11. Osuna PM, Udovcic M, Sharma MD. Hyperthyroidism and the heart. Methodist Debakey Cardiovasc J 2017;13:60-3.

12. Caulley L, Johnson-Obaseki S, Luo L, Javidnia H. Risk factors for postoperative complications in total thyroidectomy: a retrospective, risk-adjusted analysis from the National Surgical Quality Improvement Program. Medicine (Baltimore) 2017;96:e5752.

13. Brandt F, Green A, Hegedus L, Brix TH. A critical review and meta-analysis of the association between overt hyperthyroidism and mortality. Eur J Endocrinol 2011;165:491-7.

14. Costantino ME, Frey B, Hall B, Painter P. The influence of a postdischarge intervention on reducing hospital readmissions in a Medicare population. Popul Health Manag 2013; 16:310-6.

15. Ojeda S, Anguita M, Delgado M, Atienza F, Rus C, Granados AL, et al. Short- and long-term results of a programme for the prevention of readmissions and mortality in patients with heart failure: are effects maintained after stopping the programme? Eur J Heart Fail 2005;7:921-6. 